\title{
Visual and anatomical outcomes associated with treat-and-extend administration of intravitreal aflibercept for neovascular age-related macular degeneration
}

\author{
Mohamed Kamel Soliman ${ }^{1,2}$, Nicolas Tuli ${ }^{3}$, Thomas K. Lee ${ }^{1,4}$, William A. Britton ${ }^{1,4}$ and Raman Tuli ${ }^{1,4,5^{*}}$
}

\begin{abstract}
Purpose: To investigate the visual and anatomical outcomes associated with treat-and-extend (TAE) regimen of intravitreal (IVT) aflibercept in eyes with treatment naïve neovascular age-related macular degeneration (nvAMD).

Methods: A retrospective chart review of eyes that underwent IVT aflibercept injections for nvAMD between May 2014 and March 2018 was performed. The primary outcome was the change in best corrected visual acuity (BCVA) at 12 months. Secondary outcomes included the change in central retinal thickness (CRT), subretinal fluid (SRF) and intraretinal fluid (IRF).

Results: Data from 213 eyes of 213 patients (138 female, 65\%) met the inclusion criteria. The mean (SD) age of the patients was $80.4( \pm 9.2)$ years. The mean baseline BCVA $(0.92 \pm 0.50 \log M A R$, improved by $0.20( \pm 0.40)$ logMAR units at 12 months $(p<0.001)$. Seventy-two (34\%) eyes gained $\geq 0.3$ logMAR and $47(22 \%)$ eyes achieved BCVA $\leq$ $0.3 \log M A R$ at 12 months. Baseline BCVA, patient age, and the number of aflibercept injections received were predictors of the change in BCVA at 12 months. Mean CRT improved from $347( \pm 117) \mu \mathrm{m}$ at baseline to $246( \pm 55) \mu \mathrm{m}$ at 12 months ( $p$ < 0.001). The percentage of eyes with SRF and IRF on SD-OCT declined from 63 to $21 \%$ and from 60 to $26 \%$ at 12 months, respectively.

Conclusion: A TAE regimen of IVT aflibercept in treatment naïve nVAMD is associated with good visual and anatomical outcomes in routine clinical practice. Resolution of exudation occurred in about half of nvAMD cases at 12 months. Individualized administration of IVT aflibercept may reduce injection burden.
\end{abstract}

Keywords: Aflibercept, VEGF Trap-eye, Anti-VEGF, Treat-and-extend, Age-related macular degeneration

\section{Introduction}

Aflibercept or vascular endothelial growth factor (VEGF) Trap-eye (Eylea; Regeneron Pharmaceuticals), is a VEGFbinding recombinant fusion protein that demonstrated stronger VEGF binding affinity than antibody-mediated

*Correspondence: rtuli@toh.ca

${ }^{5}$ The Ottawa Hospital Riverside Campus, University of Ottawa, Ottawa, ON K1H 7W9, Canada

Full list of author information is available at the end of the article
VEGF inhibition (i.e., ranibizumab and bevacizumab) [1] and down-regulates plasma von Willebrand factor [2].

In the VIEW 1 and VIEW 2 trials, intravitreal (IVT) administration of aflibercept $(2 \mathrm{mg})$ at 1 - and 2-month intervals (following three monthly injections) was associated with significant visual improvement in patients with neovascular age-related macular degeneration (nvAMD) [3]. While effective, fixed injection schedules are less popular in clinical setting as it preclude lengthening of the therapeutic interval in eyes that require less frequent 
treatment. The majority of retina specialists in the United States (86.21\%) surveyed by The American Society of Retina Specialists (ASRS) in 2019 adopted a treat-and-extend (TAE) regimen for anti-VEGF injections [4]. The regimen involves a loading phase of 1-3 monthly anti-VEGF injections with subsequent injections interval shortened or lengthened based on the presence or absence of signs of activity of the nvAMD, respectively. Data from prospective randomized clinical studies have suggested that TAE dosing of aflibercept may enable comparable visual and anatomical improvements to those observed with fixed dosing $[5,6]$. However, as patient populations and treatment scheduling in the clinical setting may differ from a strictly controlled environment in prospective trials, the "real-world" outcomes are important to guide clinicians' expectations of treatment. To date, there is a scarcity of studies reporting the treatment outcome of aflibercept using TAE dosing in real world setting. In addition, the visual outcomes and frequency of injections associated with TAE aflibercept dosing varies widely between these studies. Perhaps this variation stems from differences in sample size, baseline characteristics and treatment criteria among different studies [7-16]. With this in mind, we conducted the current study surveying a large sample of eyes with treatment naïve nvAMD to evaluate the visual and anatomical outcomes of IVT aflibercept using a TAE protocol in routine clinical setting.

\section{Materials and methods Study design}

A retrospective chart review of all patients diagnosed with nvAMD and receiving IVT aflibercept injections between May 2014 and March 2018 was conducted. The study was conducted in the Retina Center of Ottawa, Ottawa, Ontario, Canada. Data extracted included: age, sex, best corrected visual acuity (BCVA), central retinal thickness (CRT), number of aflibercept injections, and the incidence of subretinal fluid (SRF) and intraretinal fluid (IRF) on spectral domain optical coherence tomography (SD-OCT). Patients with pre-existing macular pathology other than nvAMD, history of previous antiVEGF injection, or follow-up less than 12 months were excluded. In patients receiving IVT aflibercept bilaterally, data from either the left or right eye was randomly excluded using a randomized sequence generator. All eyes with treatment naïve nvAMD who fulfilled the above criteria during the study period were included in the analysis. Data was collected in accordance with the tenets of the Declaration of Helsinki and approved by the research ethics board.

The primary outcome measure of the study was the change in BCVA at 12 months following initiation of aflibercept therapy. Secondary outcome measures included the proportion of eyes that improved by $\geq 0.3$ logarithm of the minimal angle of resolution (logMAR) units or 15 Early Treatment Diabetic Retinopathy Study (ETDRS) letters, the proportion of eyes that achieved final VA of $\leq 0.3 \log$ MAR or $\geq 70$ ETDRS letters, as well as the change in CRT and prevalence of SRF and IRF on SD-OCT throughout the course of treatment.

\section{Injection details}

Intravitreal injections were administered monthly for the initial 3-months. The interval between subsequent injections was lengthened sequentially by $1-2$ weeks (to a maximum of 12 weeks) if SD-OCT and fundoscopy did not show signs of active exudation (i.e., complete resolution of IRF or SRF on SD-OCT). If recurrent exudation or significant visual loss ( $\geq$ Snellen line) occurs, the treatment interval was shortened by $1-2$ weeks and thereafter lengthened again if signs of exudation resolved.

\section{Data analysis}

BCVA was collected in Snellen units and converted to $\log$ MAR values and ETDRS letters for statistical analysis and comparison to previous studies [17, 18]. Data are presented as means \pm standard deviation. Repeated measures analysis of variance and post-hoc Tukey testing were performed to assess the change in BCVA and CRT between study time points. Multivariate linear regression was performed to determine factors predicting the change in BCVA from baseline to 12 months. Predictor variables for multivariate regression were selected based on plausibility of physiologically influencing a change in visual acuity. All statistical analyses and visualizations were generated using R Studio (Version 1.1.419), an integrated development environment for $\mathrm{R}$ (Version 3.5.0). A $\mathrm{p}$-value threshold of 0.05 was selected to indicate statistical significance.

\section{Results \\ Baseline characteristics}

A total of 391 eyes with nvAMD was reviewed. We initially excluded data from 15 eyes due to a history of previous anti-VEGF injections and those who received intravitreal injections other than Aflibercept (116 eyes). A further 18 eyes were excluded in patients receiving bilateral injections (Fig. 1). Data from 213 eyes of 213 patients (138 female, 65\%) met the inclusion criteria. The mean age of cohort patients was $80.4 \pm 9.2$ years. Baseline BCVA was $0.92 \pm 0.50 \operatorname{logMAR}(38.9 \pm 24.9$ ETDRS letters) and baseline CRT was $347 \pm 117 \mu \mathrm{m}$.

\section{Functional and anatomical outcomes}

The mean number of aflibercept injection was 4.4 \pm 0.7 and $7.4 \pm 1.3$ at 6 and 12 months, respectively. 


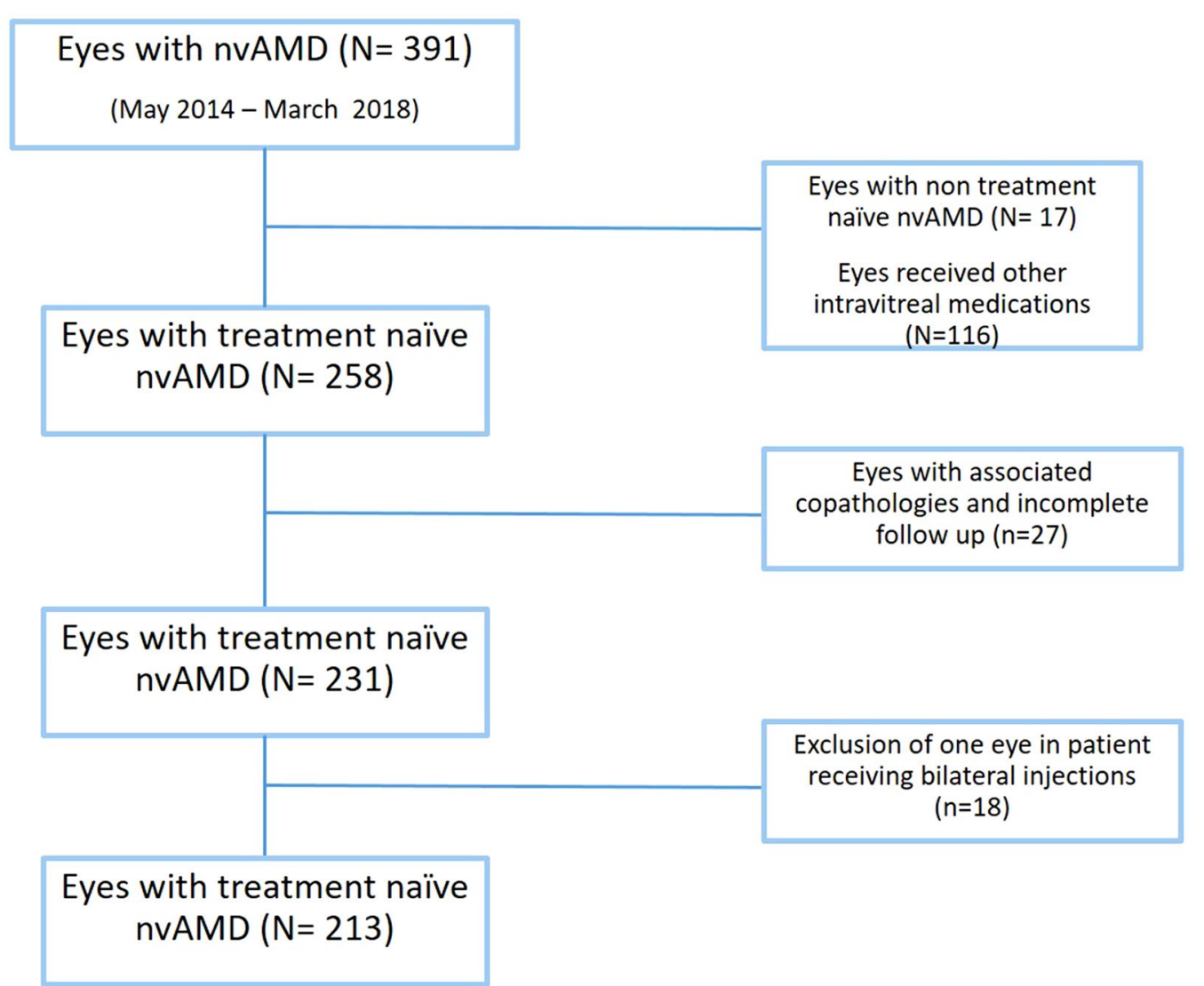

Fig. 1 A flowchart showing the number of eyes and the filtration process conducted in this study

Functional and anatomical outcomes of aflibercept injections are presented in Table 1. Mean BCVA improved from $0.92 \pm 0.50 \operatorname{logMAR}(38.9 \pm 24.9$ ETDRS letters) at baseline to $0.74 \pm 0.51 \log$ MAR
(47.8 \pm 25.3 letters $)(p<0.001)$ at 6 months. Visual gain achieved at 6 months was maintained at 12 months $(0.71 \pm 0.50 \operatorname{logMAR}, 49.3 \pm 24.9$ ETDRS letters) $(p<0.001)$ (Fig. 2A). Mean BCVA did not differ

Table 1 Functional outcomes of eyes with treatment naïve neovascular age-related macular degeneration

\begin{tabular}{ll}
\hline Baseline BCVA, mean \pm SD & $\mathbf{0 . 9 2} \pm \mathbf{0 . 5 0 ~ l o g M A R}$ (39.0 $\pm \mathbf{2 4 . 8}$ ETDRS letters) \\
\hline Post-treatment BCVA, mean \pm SD & \\
At 6 months & $0.74 \pm 0.51$ logMAR (47.9 \pm 25.3 ETDRS letters) \\
At 12 months & $0.71 \pm 0.50$ logMAR (49.3 \pm 24.9 ETDRS letters) \\
Mean change in BCVA, mean $\pm S D$ & $-0.17 \pm 0.40$ logMAR (+8.7 \pm 20.2 ETDRS letters) \\
At 6 months & $-0.20 \pm 0.40$ logMAR (+ $10.1 \pm 20.1$ ETDRS letters) \\
At 12 months & $67(31 \%)$ \\
Eyes gaining $\geq 0.3$ logMAR units (15 ETDRS letters, 3 Snellen lines), $n(\%)$ & $72(34 \%)$ \\
At 6 months & \\
At 12 months & $39(18 \%)$ \\
Eyes with $\leq 0.3$ logMAR vision ( $\geq 70$ ETDRS letters, Snellen VA $\geq 20 / 40), n(\%)$ & $47(22 \%)$ \\
At 6 months & \\
At 12 months &
\end{tabular}



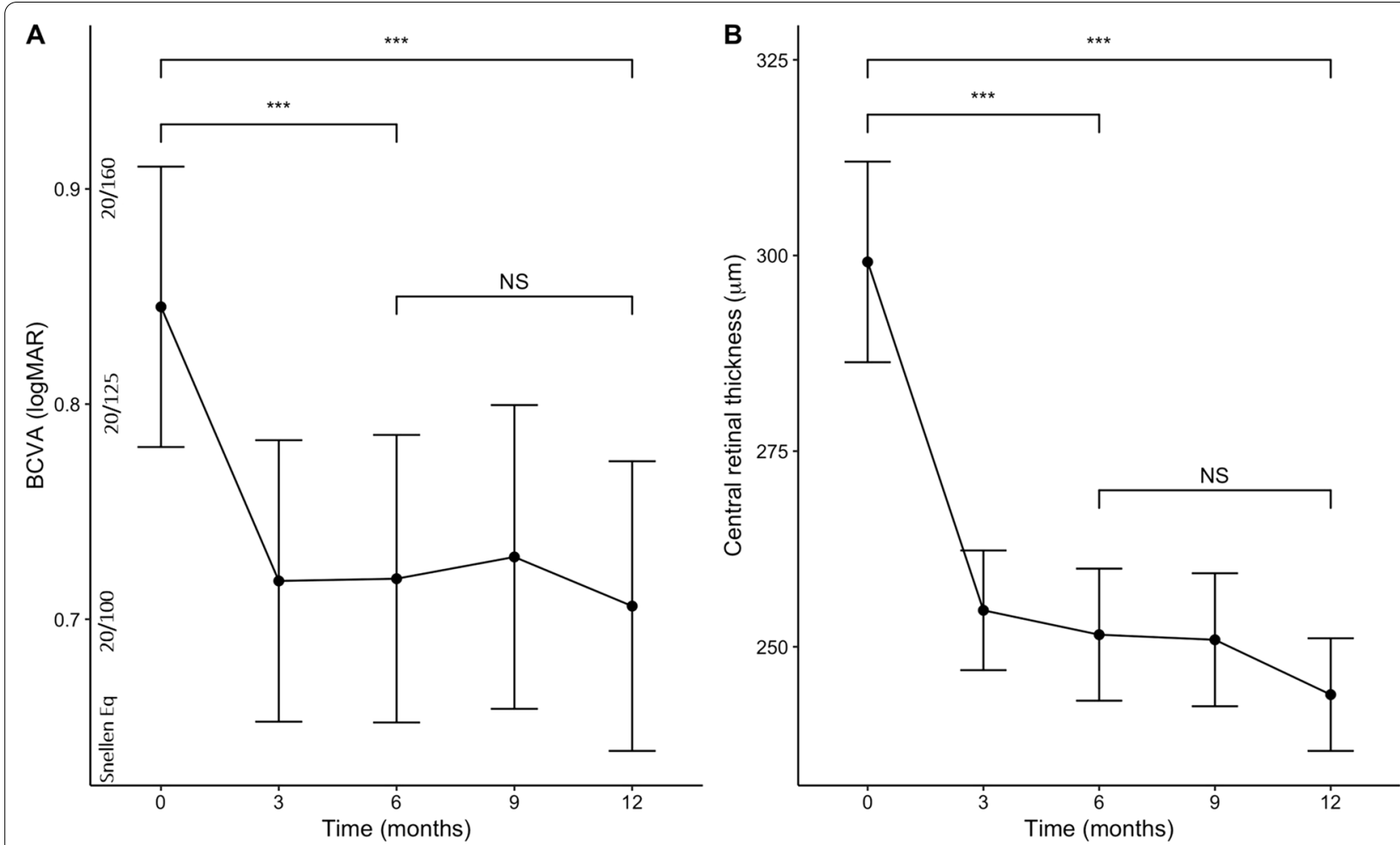

Fig. 2 Mean best-corrected visual acuity (BCVA) as logarithm of the minimal angle of resolution units $(\mathbf{A})$ and central retinal thickness $(\mathbf{B})$ as a function of intravitreal aflibercept treatment duration. Errors bars correspond to 95\% confidence intervals that extend two standard errors above and below the mean. $p<0.001\left(^{* * *}\right), p<0.01\left(^{(*}\right), p<0.05\left(^{*}\right)$, non-significant (NS)

between 6 and 12 month time points $(p=0.47)$. The number of eyes whose visual gain equalled or surpassed $0.3 \operatorname{logMAR}(+15$ ETDRS letters) was $67(31 \%)$ and $72(34 \%)$ at 6 and 12 months, respectively. At 6 and 12 months, 39 (18\%) and $47(22 \%)$ eyes, respectively, achieved BCVA $\leq 0.3 \operatorname{logMAR}$. Mean CRT decreased from $347 \pm 117 \mu \mathrm{m}$ at baseline to $254 \pm 68 \mu \mathrm{m}$ at 6 months $(p<0.001)$ and to $246 \pm 55$ at 12 months $(p<0.001)$ (Fig. 2B). Mean CRT did not differ between 6 and 12 month time points $(p=0.42)$. The proportion of eyes with SRF and IRF on SD-OCT declined from $63 \%(\mathrm{n}=134)$ to $21 \%(\mathrm{n}=45)$, and from $60 \%(\mathrm{n}$ $=128)$ to $26 \%(\mathrm{n}=55)$ at 12 months, respectively. The proportion of eyes with either SRF or IRF on SD-OCT declined from $89 \%(n=189)$ to $42 \%(n=89)$ (Fig. 3$)$. The proportion of eyes who had shortening of their treatment interval was $31 \%$ (66 eyes).

A multiple linear regression model with predictor variables including patient age, baseline BCVA and CRT significantly predicted the change in BCVA at 12 months following initiation of aflibercept therapy $\left[F_{(6,198)}=8.6\right.$, $\left.p<0.001, \mathrm{R}^{2}=0.21\right]$. Baseline BCVA $(\mathrm{B}=-0.42, p$ $<0.001)$, patient age $(\beta=0.16, p=0.019)$, and number of injections $(B=-0.16, p=0.022)$ were found to be significant predictors of the change in BCVA at 12 months (Table 2).

Data from a total of 1584 injections were analyzed in the current study. We did not observe any case of endophthalmitis or other serious, adverse complications.

\section{Discussion}

We report the 12 month functional and anatomical outcomes associated with TAE administration of intravitreal aflibercept for nvAMD in a real-world setting. To the best of our knowledge, this is the largest retrospective study to evaluate TAE aflibercept in eyes with treatment naïve nvAMD. Our findings demonstrate a mean visual improvement of $0.21 \log$ MAR units $(+10.1$ ETDRS letters) with an average of 7.4 injections amongst 213 treatment-naïve eyes with nvAMD at 12 months follow up. In addition, SD-OCT demonstrated a mean reduction of CRT by $101 \mu \mathrm{m}$ and reduced prevalence of SRF and IRF at 12 month follow-up.

To date, the Japanese Treat and Extend Study of Aflibercept in Neovascular Age-related Macular Degeneration (ALTAIR) study is the largest prospective randomized study of TAE aflibercept administration in patients with nvAMD [19]. The study compared the 


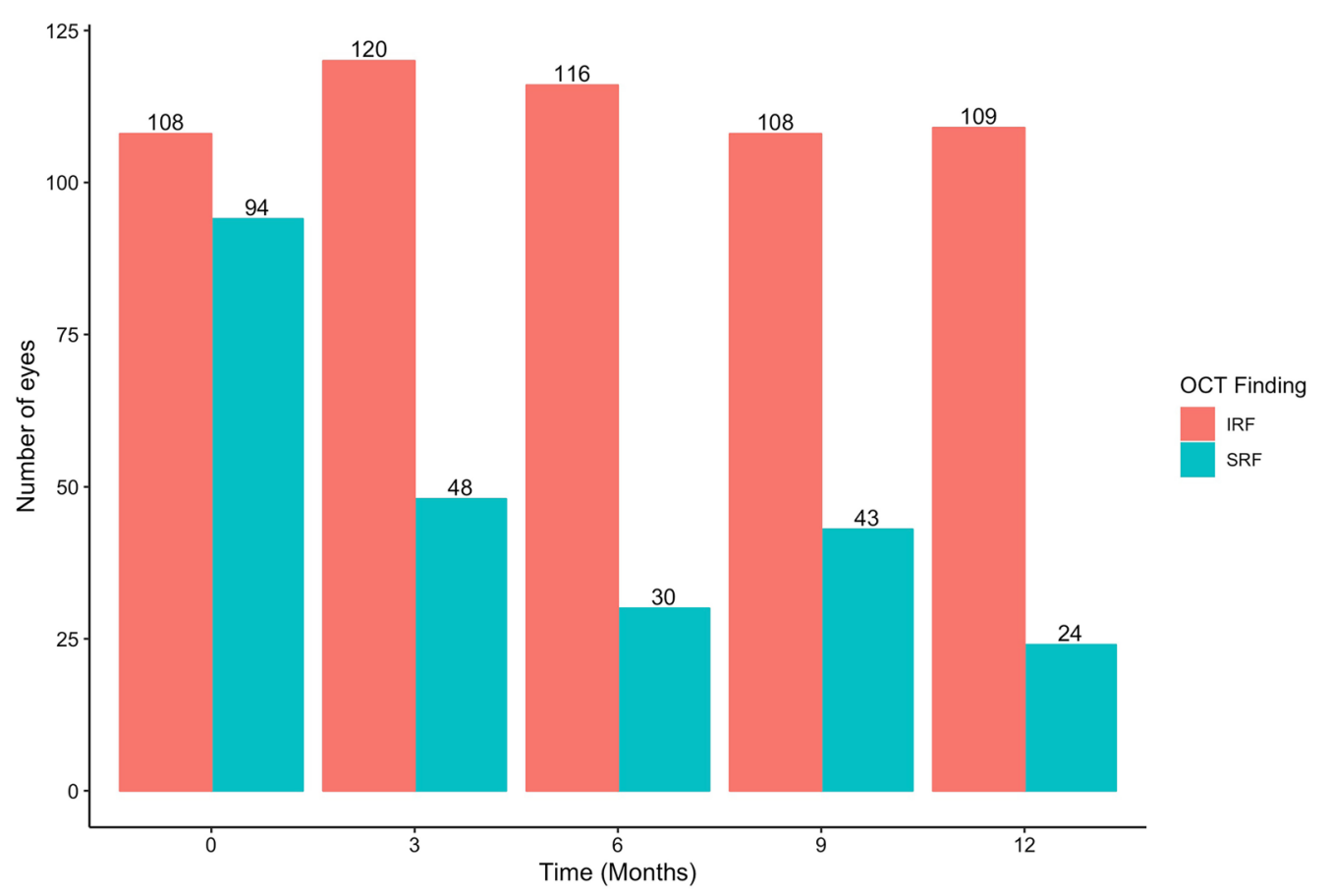

Fig. 3 Number of eyes with either subretinal fluid (SRF) or intraretinal fluid (IRF), SRF only, or IRF only on optical coherence tomography at baseline and following 12 months of intravitreal aflibercept treatment

Table 2 Results of multivariate linear regression for factors predicting the change in best corrected visual acuity (BCVA) in logMAR units at 12 months

\begin{tabular}{lll}
\hline Predictor & B & p value \\
\hline Age & 0.16 & $0.019\left(^{*}\right)$ \\
Baseline BCVA & -0.42 & $9.43 \mathrm{e}-0\left(^{* * *}\right)$ \\
Baseline CRT & -0.0063 & 0.93 \\
Number of injections & -0.16 & $0.022\left(^{*}\right)$ \\
Presence of SRF & 0.014 & 0.84 \\
Presence of IRF & -0.044 & 0.54 \\
\hline
\end{tabular}

$B C V A$ best corrected visual acuity; CRT central retinal thickness; SRF subretinal fluid; $I R F$ intraretinal fluid

$\left.p<0.0011^{* * *}\right), p<0.01{ }^{(*)}, p<0.05^{(*)}$

outcomes associated with 2 and 4 week extensions of the injection interval in eyes with nvAMD. Amongst 246 eyes, treatment extensions of 2 and 4 weeks resulted in 9.0 and 8.4 ETDRS letter gain with 7.2 and 6.9 injections at 1-year, respectively. Similarly, DeCroos et al. [5] found that a mean of 8.0 injections of TAE aflibercept resulted in a visual gain of 7.2 letters amongst 35 eyes in a prospective trial at 1-year follow-up. Haga et al. [6] observed a more significant visual gain of $0.32 \operatorname{logMAR}(\sim 15.9$ letters) from 21 eyes in a randomized to TAE aflibercept injections. In contrast, Hatz et al. and Jørstad et al. [20,
21] reported stabilization of vision following 1 year of aflibercept therapy in cohorts of eyes that had a limited response to prior anti-VEGF therapy.

Real world studies of TAE aflibercept reported widely variable visual gains ranging from 4 to 15.9 letters with 4.5-8.3 injections at 1-year (Table 3). The largest study was performed by Barthelmes and associates, including 136 treatment naïve eyes treated with TAE aflibercept. The study reported a visual gain of 6.0 ETDRS letters with 13.6 injections at 2-years follow-up [7]. In our study, we observed a greater mean visual gain (+ 10.1 ETDRS letters) relative to Barthelmes et al. which may be due to the relatively poorer baseline visual acuity of our study cohort (40.0 vs. 61.4 ETDRS letters). In contrast, Unsal et al. and Castro-Navarro et al. [13, 14] reported greater mean visual gains of 15.9 and 11.5 ETDRS letters with mean of 4.9 and 7.7 injections, respectively, at 1 year. However, the latter studies included a small number of eyes, making it difficult to draw firm conclusions about the expected visual gain. Recently, a study analyzing the Intelligent Research in Sight (IRIS) Registry provided the largest real world visual acuity results in patients with treatment naïve nvAMD treated with a single anti-VEGF, regardless of the treatment regimen employed (fixed, TAE, pro-re-nata) [22]. Among 4387 patients treated with aflibercept, the mean visual gain was $0.04 \log M A R$ (+ 2.0 ETDRS letter). This minimal change in VA may 
Table 3 Summary of previous studies using aflibercept for neovascular age-related macular degeneration (nvAMD) with a treat-andextend (TAE) regime

\begin{tabular}{|c|c|c|c|c|c|c|}
\hline \multicolumn{7}{|l|}{ Prospective studies } \\
\hline Study & $\mathrm{n}$ & Mean age & $\begin{array}{l}\text { Mean baseline VA } \\
\text { (ETDRS letters) }\end{array}$ & $\begin{array}{l}\text { Visual change } \\
\text { (ETDRS letters) }\end{array}$ & $\begin{array}{l}\text { Number of } \\
\text { injections }\end{array}$ & Treatment näive \\
\hline \multirow[t]{2}{*}{ Ohji et al. (ALTAIR) [19] } & $124^{\mathrm{a}}$ & NA & NA & $+9.0(1 \mathrm{y})$ & 7.2 & \multirow[t]{2}{*}{ Yes } \\
\hline & $123^{b}$ & NA & NA & $+8.4(1 \mathrm{y})$ & 6.9 & \\
\hline Haga et al. [6] & 21 & 75.5 & 56.9 & $+15.9(1 y)$ & 7.5 & NR \\
\hline DeCroos et al. (ATLAS) [5] & 35 & 81.3 & 58.9 & $+7.2(1 \mathrm{y})$ & 8.0 & Yes \\
\hline Hatz et al. [20] & 33 & 81.0 & 66.8 & $-0.6(1 \mathrm{y})$ & NR & No \\
\hline Jørstad et al. [21] & 50 & NR & $72.5^{9}$ & $+0.5(1 y)^{9}$ & 9.2 & No \\
\hline \multicolumn{7}{|l|}{ Retrospective studies } \\
\hline Study & $\mathbf{n}$ & Mean age & $\begin{array}{l}\text { Mean baseline VA } \\
\text { (ETDRS letters) }\end{array}$ & $\begin{array}{l}\text { Mean visual gain } \\
\text { (ETDRS letters) }\end{array}$ & $\begin{array}{l}\text { Number of } \\
\text { injections }\end{array}$ & Treatment näive \\
\hline Barthelmes et al. [7] & 136 & 77.2 & 61.4 & $+6.0(2 y)$ & 13.6 & Yes \\
\hline \multirow[t]{4}{*}{ Matsumoto et al. [8] } & $18^{\mathrm{c}}$ & 75.7 & $53.5^{9}$ & $+9.5(1 y)^{9}$ & 7.7 & Yes \\
\hline & $44^{d}$ & 71.5 & $72.0^{9}$ & $+7.0(1 y)^{9}$ & 8.3 & Yes \\
\hline & $58^{e}$ & 72.4 & $71.5^{\mathrm{g}}$ & $+8.0(1 y)^{g}$ & 7.7 & Yes \\
\hline & $5^{f}$ & 77.2 & $56.5^{9}$ & $+14.5(1 y)^{9}$ & 7.3 & Yes \\
\hline Matsumoto et al. [9] & 60 & 75.1 & $69.5^{9}$ & $+9.0(1 y)^{9}$ & $13.8(2 \mathrm{y})$ & Yes \\
\hline Ohnaka et al. [10] & 36 & 72.3 & $61.0^{9}$ & $+4(1 y)^{9}$ & 4.5 & Yes \\
\hline Yamamoto et al. [11] & 67 & NA & $70.5^{9}$ & $+7.5(1 y)^{9}$ & 8.3 & NA \\
\hline Ito et al. [12] & 61 & NA & NA & $+6.5(2 y)^{9}$ & 13.6 & Yes \\
\hline Castro-Navarro et al. [13] & 30 & 78.8 & $54.5^{9}$ & $+11.5(1 y)^{9}$ & 7.7 & Yes \\
\hline Unsal et al. [14] & 38 & 74.5 & 39.1 & $+15.9(1 y)$ & 4.9 & Yes \\
\hline Ishibashi et al. [15] & 39 & 75.5 & 63.9 & $+6.5(1 \mathrm{y})$ & 7.9 & Mixed \\
\hline Wakuta et al. [16] & 16 & 78.3 & 65 & $0(1 \mathrm{y})$ & $7.8(1 \mathrm{y})$ & Mixed \\
\hline Current Study & 213 & 80.4 & 40.0 & $+10.1(1 y)$ & 7.4 & Yes \\
\hline
\end{tabular}

ETDRS Early Treatment Diabetic Retinopathy Study; VA visual acuity; NR not reported; NA not available; $1 y$ one year; $2 y$ two year

a 2-week adjustment of treat-and-extend regime

${ }^{\mathrm{b}}$ 4-week adjustment of treat-and-extend regime

${ }^{c}$ Classic (type I) choroidal neovascularization

d Occult (type I) choroidal neovascularization

e $P C V$ polypoidal choroidal vasculopathy

${ }^{f} R A P$ retinal angiomatous proliferation

${ }^{g}$ Values reported in logarithm of the minimal angle (logMAR) and converted to approximate ETDRS letters for table

be explained by the better baseline VA (0.53 $\log M A R)$ of the IRIS cohort, suggesting a reduced potential to visual improvement. The TAE protocol in the current study resulted in visual outcomes that compared well with the results obtained in VIEW1 and VIEW2 clinical trials but with less frequent injections. In VIEW1 and VIEW2, intravitreal aflibercept given monthly (2q4) improved baseline vision by 10.9 and 7.6 ETDRS letters at 1-year in 304 and 309 eyes, respectively [3]. Although, inter-study comparison is limited by variability in patient characteristics, associated copathologies and lesion size, the visual gain in the current study is similar to those reported in prospective clinical trials and falls within the range reported by previous retrospective TAE aflibercept studies (Table 3).

We found worse baseline visual acuity, increased number of injections, and decreased patient age to significantly predict visual gain in our study (Table 2). Worse baseline vision was previously associated with larger changes in visual acuity in patients receiving nvAMD treatment [23].

Improvement of SRF and IRF over time is an important measure of anti-VEGF efficacy. With fixed, monthly (2q4) dosing in the VIEW1 and VIEW2 trials, the incidence of eyes with SRF or IRF declined from 100\% at baseline to $35.2 \%$ and $19.7 \%$ over 1 -year, respectively [3]. With TAE 
dosing, Jørstad et al. [21] reported a $44 \%$ decline in the prevalence of eyes with SRF or IRF over 1-year while Hatz et al. [20] reported a reduction of $44.8 \%$ over 2 -years. The number of eyes with SRF or IRF in our cohort similarly declined by $47 \%$ over 12 months.

Limitations of the current study include its retrospective design, lack of comparative control group and relatively short-term follow-up. In addition, due to the retrospective nature of the study, it is challenging to determine the degree of adherence to protocol and whether all planned injections were given within the proper time frame. Furthermore, conversion of VA values from Snellen to EDTRS scoring system may affect the accuracy of the results. However, our study is strengthened by several factors. To the best of our knowledge, our cohort represents the largest sample of treatment naïve nvAMD eyes treated with TAE aflibercept in routine clinical practice. In addition, eyes were not excluded on the basis of severity of nvAMD. Our data therefore reflects real-world clinical outcomes of TAE aflibercept administration in treatment-naïve eyes at 12 months follow-up.

\section{Conclusion}

In conclusion, our results demonstrated that TAE administration of intravitreal aflibercept in real-world clinical practice improves vision and anatomical correlates in eyes with treatment naïve nvAMD. Treat-and-extend administration may minimize injection burden and healthcare expenditure without negatively impacting functional outcomes. Our results may help to guide clinicians about treatment expectations in less strictly controlled clinical environments.

\begin{abstract}
Abbreviations
ASRS: American Society of Retina Specialists; BCVA: Best corrected visual acuity; CRT: Central retinal thickness; ETDRS: Early Treatment Diabetic Retinopathy Study; nvAMD: Neovascular age-related macular degeneration; IRIS: Intelligent Research in Sight Registry; IRF: Intraretinal fluid; IVT: Intravitreal; logMAR: Logarithm of the minimal angle of resolution; SE: Snellen equivalent; SRF: Subretinal fluid; SD: Standard deviation; SD-OCT: Spectral domain optical coherence tomography; TAE: Treat-and-extend regimen; VEGF: Vascular endothelial growth factor; VA: Visual acuity.
\end{abstract}

\section{Acknowledgements}

None.

\section{Authors' contributions}

MKS: conception and design, drafting manuscript, final review of the manuscript. NT: data collection, drafting manuscript. TL: conception and design, data gathering. WB: conception and design, data gathering. RT: conception and design, data gathering, final review of the manuscript. All authors read and approved the final manuscript.

\section{Funding}

None.

Availability of data and materials

Data are available on demand.

\section{Declarations}

\section{Ethics approval and consent to participate}

Data was collected in accordance with the tenets of the Declaration of Helsinki and approved by the research ethics board. Consent was not required due to the retrospective nature of the study.

\section{Consent for publication}

The paper was approved for publication.

\section{Competing interests}

The authors declare that they have no competing interests.

\section{Author details}

${ }^{1}$ Department of Ophthalmology, University of Ottawa, Ottawa, ON, Canada. ${ }^{2}$ Department of Ophthalmology, Faculty of Medicine, Assiut University, Assiut, Egypt. ${ }^{3}$ Faculty of Arts and Science, University of Toronto, Toronto, ON, Canada. ${ }^{4}$ Retina Center of Ottawa, Ottawa, ON, Canada. ${ }^{5}$ The Ottawa Hospital Riverside Campus, University of Ottawa, Ottawa, ON K1H 7W9, Canada.

Received: 9 January 2021 Accepted: 11 September 2021

Published online: 27 September 2021

\section{References}

1. Holash J, Davis S, Papadopoulos N, Croll SD, Ho L, Russell M, et al. VEGFTrap: a VEGF blocker with potent antitumor effects. Proc Natl Acad Sci. 2002;99(17):11393-8.

2. Yamashita M, Matsumoto M, Hayakawa M, Sakai K, Fujimura Y, Ogata NJSR. Intravitreal injection of aflibercept, an anti-VEGF antagonist, downregulates plasma von Willebrand factor in patients with age-related macular degeneration. Sci Rep. 2018;8(1):1491.

3. Heier JS, Brown DM, Chong V, Korobelnik J-F, Kaiser PK, Nguyen QD, et al. Intravitreal aflibercept (VEGF trap-eye) in wet age-related macular degeneration. Ophthalmology. 2012;119(12):2537-48.

4. America Society of Retina Specialists Preferences and Trends Survey. 2021. https://www.asrs.org/content/documents/_asrs-2019-pat-surveyresults-for-website2.pdf. Accessed 08 July 2021.

5. DeCroos FC, Reed D, Adam MK, Salz D, Gupta OP, Ho AC, et al. Treatand-extend therapy using aflibercept for neovascular age-related macular degeneration: a prospective clinical trial. BMC Ophthalmol. 2017:180:142-50.

6. Haga A, Kawaji T, Ideta R, Inomata Y, Tanihara HJAO. Treat-and-extend versus every-other-month regimens with aflibercept in age-related macular degeneration. Acta Ophthalmol. 2018;96(3):e393-8.

7. Barthelmes D, Nguyen V, Daien V, Campain A, Walton R, Guymer R, et al. Two year outcomes of "treat and extend" intravitreal therapy using aflibercept preferentially for neovascular age-related macular degeneration. Retina. 2018:38(1):20-8.

8. Matsumoto H, Morimoto M, Mimura K, Ito A, Akiyama HJOR. Treat-andextend regimen with aflibercept for neovascular age-related macular degeneration: efficacy and macular atrophy development. Ophthalmol Retin. 2018;2(5):462-8.

9. Matsumoto H, Hiroe T, Morimoto M, Mimura K, Ito A, Akiyama H. Efficacy of treat-and-extend regimen with aflibercept for pachychoroid neovasculopathy and Type 1 neovascular age-related macular degeneration. Jpn J Ophthalmol. 2018;62(2):144-50.

10. Ohnaka M, Nagai Y, Sho K, Miki K, Kimura M, Chihara T, et al. A modified treat-and-extend regimen of aflibercept for treatment-naïve patients with neovascular age-related macular degeneration. Graefe's Arch Clin Exp Ophthalmol. 2017;255(4):657-64.

11. Yamamoto A, Okada AA, Nakayama M, Yoshida Y, Kobayashi HJO. One-year outcomes of a treat-and-extend regimen of aflibercept for exudative age-related macular degeneration. Ophthalmologica. 2017;237(3):139-44

12. Ito A, Matsumoto H, Morimoto M, Mimura K, Akiyama HJO. Two-year outcomes of a treat-and-extend regimen using intravitreal aflibercept injections for typical age-related macular degeneration. Ophthalmologica. 2017;238(4):236-42. 
13. Castro-Navarro V, Cervera-Taulet E, Montero-Hernández J, Navarro-Palop C. Estrategia «Tratar y Extender» con aflibercept: efecto en diferentes tipos de neovascularización coroidea asociada a la edad. Arch Soc Esp Oftalmol. 2017;92(3):112-9.

14. Unsal E, Cubuk MO. The results of aflibercept therapy as a first line treatment of age-related macular degeneration. J Curr Ophthalmol. 2019;31(1):66-71.

15. Ishibashi K, Haruta M, Ishibashi Y, Noda R, Dake S, Yoshida S. Four-year outcomes of intravitreal aflibercept treatment for neovascular age-related macular degeneration using a treat-and-extend regimen in Japanese patients. Ther Adv Ophthalmol. 2021;13:2515841420984586. https://doi. org/10.1177/2515841420984586.

16. Wakuta M, Nomi N, Ogata T, Ota M, Yamashiro C, Hatano M, et al. A Trinity regimen with aflibercept for treatment-naïve neovascular age-related macular degeneration: 2-year outcomes. Graefe's Arch Clin Exp Ophthalmol. 2020;258(8):1663-70. https://doi.org/10.1007/s00417-020-04745-1.

17. Beck RW, Moke PS, Turpin AH, Ferris FL III, SanGiovanni JP, Johnson CA, et al. A computerized method of visual acuity testing: adaptation of the early treatment of diabetic retinopathy study testing protocol. Am J Ophthalmol. 2003;135(2):194-205.

18. Gregori NZ, Feuer W, Rosenfeld PJ. Novel method for analyzing snellen visual acuity measurements. Retina. 2010;30(7):1046-50. https://doi.org/ 10.1097/IAE.0b013e3181d87e04

19. Ohji M, Okada A, Takahashi K, Kobayashi M, Terano Y. Two different treat and extend dosing regimens of intravitreal aflibercept for WAMD in
Japanese patients: 52 week results of the ALTAIR Study. In: Presentation at the 17th European Society of Retina Specialists (EURETINA) Congress on 2017. Barcelona: EURETINA Congress; 2017.

20. Hatz K, Prünte CJR. Intravitreal aflibercept in neovascular age-related macular degeneration with limited response to ranibizumab: a treat-andextend trial. Retina. 2017;37(6):1185-92.

21. Jørstad ØK, Faber RT, Moe MC. Two-year functional and anatomical results after converting treatment resistant eyes with exudative agerelated macular degeneration to aflibercept in accordance with a treat and extend protocol. Acta ophthalmol. 2017;95(5):460-3.

22. Rao P, Lum F, Wood K, Salman C, Burugapalli B, Hall R, et al. Real-world vision in age-related macular degeneration patients treated with single anti-VEGF drug type for 1 year in the IRIS registry. Ophthalmology. 2018;125(4):522-8.

23. Kikushima W, Sakurada Y, Sugiyama A, Yoneyama S, Tanabe N, Matsubara $\mathrm{M}$, et al. Comparison of two-year outcomes after photodynamic therapy with ranibizumab or aflibercept for polypoidal choroidal vasculopathy. Sci Rep. 2017;7(1):16461.

\section{Publisher's Note}

Springer Nature remains neutral with regard to jurisdictional claims in published maps and institutional affiliations.
Ready to submit your research? Choose BMC and benefit from:

- fast, convenient online submission

- thorough peer review by experienced researchers in your field

- rapid publication on acceptance

- support for research data, including large and complex data types

- gold Open Access which fosters wider collaboration and increased citations

- maximum visibility for your research: over 100M website views per year

At BMC, research is always in progress.

Learn more biomedcentral.com/submissions 